\title{
Decreased central fatigue in multiple sclerosis patients after 8 weeks of surface functional electrical stimulation
}

\author{
Ya-Ju Chang, PhD; ${ }^{*}$ Miao-Ju Hsu, PhD; ${ }^{2}$ Shin-Man Chen, MS; ${ }^{1}$ Cheng-Hsiang Lin, PhD; ${ }^{3}$ Alice M. K. Wong, MD $^{1}$ \\ ${ }^{1}$ Department of Physical Therapy and Graduate Institute of Rehabilitation Science, Chang Gung University, Taoyuan, \\ Taiwan; ${ }^{2}$ Department of Physical Therapy, College of Health Science, Kaohsiung Medical University, and Department \\ of Physical Medicine and Rehabilitation, Kaohsiung Medical University Hospital, Kaohsiung, Taiwan; ${ }^{3}$ Department of \\ Statistics, Tunghai University, Taichung, Taiwan
}

\begin{abstract}
Effective treatments for multiple sclerosis (MS)associated central fatigue have not been established. Surface functional electrical stimulation (FES), which can challenge the peripheral neuromuscular system without overloading the central nervous system, is a relatively safe therapeutic strategy. We investigated the effect of 8 weeks of surface FES training on the levels of general, central, and peripheral fatigue in MS patients. Seven of nine individuals with MS (average age: 42.86 +/13.47 years) completed 8 weeks of quadriceps muscle surface FES training. Maximal voluntary contraction, voluntary activation level, twitch force, General Fatigue Index (FI), Central Fatigue Index (CFI), Peripheral Fatigue Index, and Modified Fatigue Impact Scale (MFIS) scores were determined before and after training. The results showed that FI $(p=0.01)$, CFI $(p=$ $0.02)$, and MFIS $(p=0.02)$ scores improved significantly after training. Improvements in central fatigue contributed significantly to improvements in general fatigue $(p<0.01)$. The results of the current study showed that central fatigue was a primary limitation in patients with MS during voluntary exercise and that 8 weeks of surface FES training for individuals with MS led to significantly reduced fatigue, particularly central fatigue.
\end{abstract}

Key words: central fatigue, demyelinating disease, fatigue, functional electrical stimulation, interpolated twitch, maximum voluntary contraction, multiple sclerosis, peripheral fatigue, rehabilitation, twitch.

\section{INTRODUCTION}

Multiple sclerosis (MS) is characterized by demyelinating lesions throughout the white matter of the central nervous system (CNS) [1]. Myelin serves as an insulator that speeds up conduction along nerve fibers from one node of Ranvier to another and conserves energy for the axon as depolarization occurs only at the nodes [2]. Patients with MS exhibit multiple impairments of the motor, sensory, and/or visual systems. Fatigue is one of the most commonly reported symptoms, and it can be a significant cause of disability.

\footnotetext{
Abbreviations: CFI $=$ Central Fatigue Index, CNS = central nervous system, EDSS = Expanded Disability Status Scale, EMG = electromyography, FES = functional electrical stimulation, FI = General Fatigue Index, GEE = generalized estimating equations, ITT $=$ interpolated twitch technique, MFIS $=$ Modified Fatigue Impact Scale, MS = multiple sclerosis, MVC = maximum voluntary contraction, $\mathrm{PFI}=$ Peripheral Fatigue Index, $\mathrm{T} 1=$ resting twitch force, $\mathrm{T} 2=$ superimposed twitch force, VA = voluntary activation level.

*Address all correspondence to Ya-Ju Chang, PhD; 259 Wen-Hwa 1st Road, Kwei-Shan, Taoyuan, Taiwan; +886-32118800, ext 5515; fax: +886-3-2118700.

Email: vichang@mail.cgu.edu.tw

DOI:10.1682/JRRD.2010.03.0038
} 
Conventionally, fatigue is classified as central or peripheral fatigue according to whether it is associated with the CNS or the peripheral neuromuscular system. Previous studies have reported that individuals with MS experienced higher levels of central fatigue relative to nondisabled individuals [3-4]. Increased central fatigue in patients with MS may be associated with impairment of neural transmission due to demyelination, which causes nerves to fatigue rapidly [1]. However, Lenman et al. found that patients with MS showed greater fatigability and greater slowing of tibialis anterior muscle relaxation after repetitive stimulation at $40 \mathrm{~Hz}$ [5], suggesting that patients with MS also experience higher levels of peripheral fatigue than nondisabled subjects. To avoid fatigue, clinicians often recommend that patients with MS avoid excessive exercise and conserve energy in their daily activities. However, long-term inactivity can further increase the severity of fatigue [6]. Without knowing the relative levels of central and peripheral fatigue, clinicians often have difficulty designing effective rehabilitation programs and evaluating the effects of treatment in individuals with MS.

Relative contributions of central and peripheral factors to fatigue have not been quantified in individuals with MS. The generalized estimating equations (GEE) model, which accounts for the correlation between observations in generalized linear regression models [7-8], is a potential method to weight the relative contributions of central and peripheral fatigue. GEE have been employed to successfully quantify the weightings of local muscle factors associated with the increase in perceived exertion during stepping exercises in nondisabled subjects [9]. In a repeated-measures design, repeat observations are correlated over time. If this correlation is not considered, the standard errors of the parameter estimates will be invalid [7-8]. Therefore, even though a multiple regression model could assign the weightings of independent variables to the predicted variable, the GEE model is more appropriate.

Currently, no therapeutic strategies have been designed specifically for patients prone to central fatigue. Previous studies have reported that patients with MS could benefit from endurance training, strength training, and aerobic exercise. Svensson et al. found that 4 to 8 weeks of resistance training designed to increase knee flexor endurance led to improved muscle strength and decreased perceptions of fatigue in three out of five subjects [10]. DeBolt and McCubbin found that 8 weeks of home-based resistance exercise training increased leg power [11]. Aerobic exercise, which has been reported to increase the isokinetic peak torque of the knee extensor [12], increased both the distance covered in a 6-minute walking test [13] and maximal oxygen consumption [1416]. However, this type of training requires active patient participation. Patients with lower functional ability and higher levels of fatigue usually fail to participate in this form of training. Furthermore, because equipment for measuring exercise intensity is often not conveniently available in clinics or at home, clinicians and their patients with MS tend to use a lower than optimal training intensity to avoid negative side effects associated with exercise, such as increased body temperature and excessive fatigue.

Identifying a safe and effective therapeutic strategy for patients with MS that can provide sufficient challenge to the neuromuscular system without overloading the CNS is essential [17]. Surface functional electric stimulation (FES), also sometimes called neuromuscular electrical stimulation, can activate muscles and bypass the CNS. It may be a promising approach. Previous studies found that FES improved the strength of patients with postradiculopathy [18] and stroke-associated muscle weakness [19] and decreased muscle fatigue in patients with chronic heart failure [20]. Studies have also reported that electrical stimulation at an intensity above the motor threshold led to increased motor cortex excitability [2122], suggesting that surface FES might also be effective in overcoming central fatigue.

The current study evaluated the effect of a surface FES training program on muscle strength and fatigability in patients with MS. The current study also quantified the relative contributions of central and peripheral fatigue to general fatigue. Furthermore, the current study determined whether the surface FES training program relieved central or peripheral fatigue. We hypothesized that 8 weeks of surface FES training on knee extensors would lead to significant improvements in maximum voluntary contraction (MVC) force, voluntary activation level (VA), twitch force, central fatigue, peripheral fatigue, general fatigue, and perceived fatigue in patients with MS.

\section{METHODS}

Nine individuals diagnosed with MS were recruited with informed consent. Subjects met the following inclusion criteria: (1) definite diagnosis of MS and stable for at 
least 4 months, (2) between 20 and 60 years old, and (3) active muscle contraction of quadriceps. Subjects were excluded if they had previous history of osteoporosis, other neuromuscular-skeletal diseases, or cardiovascular diseases or were unable to tolerate supramaximal stimulation. Manual muscle testing was performed on the knee extensors by a physical therapist. The Kurtzke Expanded Disability Status Scale (EDSS) [23] was administered by a physician. For included subjects, the strength of the knee extensors was $<$ Grade 3 and the EDSS scores ranged from 1.0 to 4.0.

Each subject sat on the Biodex system (Biodex Medical Systems; Shirley, New York) with their knee joint fixed at a $60^{\circ}$ flexion angle while we measured the isometric force of the knee extensor. During testing, surface electrodes $(9 \times 12 \mathrm{~cm})$ for electrical stimulation (stimulator model DS7A, Digitimer Ltd; Hertfordshire, England) were placed on the muscle belly of the quadriceps, with one electrode approximately $4 \mathrm{~cm}$ above the superior border of the patella and the other electrode approximately $4 \mathrm{~cm}$ below the inguinal line. The positions of the electrodes were adjusted to produce only knee extension. Large electrodes were used because the quadriceps are large muscles and the current density delivered by large electrodes would be low and tolerable for subjects. A single pulse of electrical stimulation (pulse width $=200 \mu$ s) with a supramaximal intensity was used for eliciting the twitch and superimposed twitch. The stimulation intensity was started low and was progressively increased until no further increase in twitch force was noted. This intensity was defined as maximum intensity. The intensity was then adjusted up to 110 percent of the maximum intensity, and this level was defined as supramaximal intensity. The force was monitored online by an oscilloscope, and the force signal was digitized using an analogto-digital converter with 12-bit resolution (MetraByte DAS 1600, Keithley Instruments, Inc; Cleveland, Ohio) at $1,000 \mathrm{~Hz}$.

Subjects were instructed to totally relax their hamstrings before each testing twitch. Electromyography (EMG) signals from the hamstrings were monitored on an oscilloscope but were not recorded for analysis. If subjects contracted their hamstrings, the trial was eliminated. The crosstalk of the stimulus artifact had very little influence on the observation of hamstrings EMG activities, since the artifact occurred earlier than the EMG activity.

During the pretraining test session, subjects performed five knee extension MVCs for practice and warm-up. Subjects were then instructed to not contract their hamstrings, and the resting EMG of the hamstrings was monitored. After the subject learned to perform knee extension MVCs with minimal hamstring EMG activity, three additional knee extension MVCs, each sustained for 5 seconds, were recorded.

After the MVC test, the VA of the subjects was evaluated by interpolated twitch technique (ITT). ITT is a noninvasive method for assessing the completeness of muscle activation during voluntary contractions, especially for testing whether a muscle is fully active during a MVC [24]. In addition, this methodology has been widely applied in clinical research related to central fatigue issues [25-26]. During this test, subjects were asked to relax and then perform knee extensor MVCs. Maximal twitches were elicited by supramaximal electrical stimulation of the quadriceps during relaxation, at the 2nd second during the MVC, and at the 3rd second after the MVC to obtain the unpotentiated twitch, the superimposed twitch, and the potentiated twitch, respectively. The superimposed twitch represents the forces generated from those motor units that failed to be activated by the CNS [13]. The VA could be obtained by Equation 1:

$$
\mathrm{VA}=\left(1-\frac{\mathrm{T}_{2}}{\mathrm{~T}_{1}}\right) \times 100 \%,
$$

where $\mathrm{T} 1$ = resting twitch force, which is the average of two unpotentiated and two potentiated twitches, and T2 = superimposed twitch force. This VA test was repeated twice with 5 seconds between repetitions.

Subjects were then asked to perform a fatigue exercise in which they performed knee extensor MVCs with a contraction/relaxation ratio of 5 seconds/5 seconds, which persisted for 20 minutes. The VA test was performed twice every 4 minutes and at the end of the fatigue exercise.

After the pretraining test, the subjects received 8 weeks of surface FES training. To simplify the homebased training setting, subjects sat on a chair with their legs fixed on a custom-made frame to maintain the knee at a $90^{\circ}$ flexion, rather than the $60^{\circ}$ flexion angle used during the pretraining test session. A portable electrical stimulator (Sanaform, Italy) was used to produce isometric knee extension contractions. The frequency, pulse width, and on/off time of the surface FES were set at $25 \mathrm{~Hz}, 200 \mu \mathrm{s}$, and $500 \mathrm{~ms} / 1 \mathrm{~s}$, respectively. The training was performed at subjects' homes for 8 weeks, 30 minutes a day, 3 days a week. During the first 2 weeks, the stimulation intensity 
was set at 50 percent of the intensity required to produce 40 percent MVC, which was determined previously in the laboratory. The stimulation intensity was increased weekly until the targeted training intensity required to produce 40 percent MVC was reached. Most of the subjects reached the targeted training intensity at the beginning of the third week. A physical therapist visited each of the subjects once a week to supervise the training setting, monitor the training, and adjust the intensity of the electrical stimulation. The two remaining weekly sessions were carried out with help from subjects' family members.

After 8 weeks of training, the subjects returned to the laboratory and underwent the same tests performed during the pretraining test. Before and after the 8 weeks of training, the Modified Fatigue Impact Scale (MFIS) was administered to measure perceived fatigue.

\section{Data Analysis}

The amplitude of the MVC force was calculated from the force-time curve. To avoid possible changes in forcecontraction velocity before and after the fatigue exercise, in each of MVCs we only averaged data from the peak until $0.5 \mathrm{~s}$ after the peak to determine the amplitude of the MVC force (Figure 1(a)). The amplitudes of the three consecutive MVCs were averaged for statistical analysis. The amplitude of T1 was the average of the two unpotentiated and the two potentiated twitches (Figure 1(e)) during the ITT test. To determine the VA, we first obtained the ratio of T2 (Figure 1(c)) to T1 and then calculated the VA using Equation 1 [27-28].

The quotient of the torques after a fatigue protocol is presented as the Fatigue Index, which is commonly used as an index of a muscle's ability to resist fatigue [29-30]. The decrease in MVC forces after fatigue exercise represents both peripheral and suprasegmental parts of fatigue [27-28,31]. The General Fatigue Index (FI) was thus calculated by dividing the averaged amplitude of the three MVC forces after the fatigue exercise by the averaged amplitude of the three MVC forces before the fatigue exercise (Equation 2). The decrease of the electrical elicited twitch forces after fatigue exercise represents only the peripheral part of fatigue [31]. The Peripheral Fatigue Index (PFI) was thus calculated by dividing the averaged amplitude of the T1 after the fatigue exercise by the averaged amplitude of the $\mathrm{T} 1$ before the fatigue exercise (Equation 3). The decrease of VA after fatigue exercise represents only the suprasegmental parts of fatigue [2728]. The Central Fatigue Index (CFI) was calculated by (a)

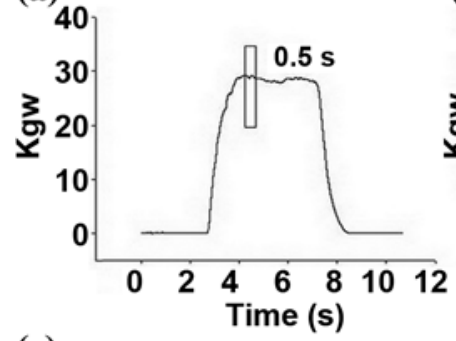

(c)

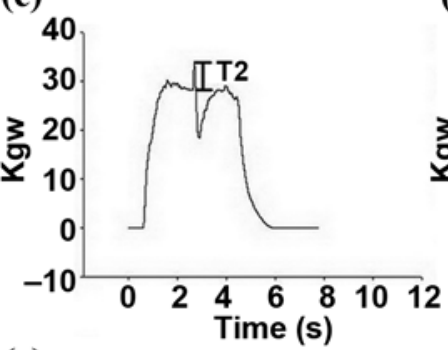

(e)

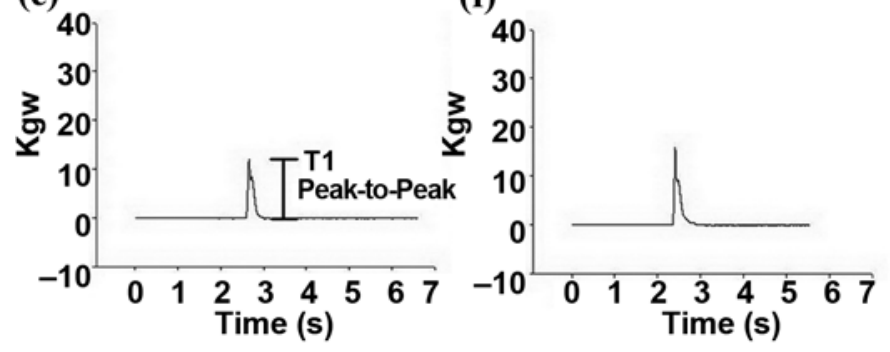

Figure 1.

Representative force-time curve during (a-b) maximum voluntary contraction (MVC), (c-d) voluntary activation level, and (e-f) unpotentiated twitch and measurement from subject before fatigue ((a), (c), (e)) and after fatigue ((b), (d), (f)). In (a), data from peak to $0.5 \mathrm{~s}$ after peak (in box) was averaged to represent amplitude of MVC force. In (c), peak-topeak amplitude represents amplitude of superimposed twitch force (T2). In (e), peak-to-peak amplitude is averaged with potentiated twitch (not shown) to represent amplitude of resting twitch force (T1).

dividing the averaged VA after the fatigue exercise by the averaged VA before the fatigue exercise (Equation 4).

$$
\begin{array}{r}
\mathrm{FI}=\frac{\frac{1}{n} \sum_{i=1}^{n} \mathrm{MVC}_{\text {postfatigue }}}{\frac{1}{n} \sum_{i=1}^{n} \mathrm{MVC}_{\text {prefatigue }}}, \\
\text { PFI }=\frac{\frac{1}{n} \sum_{i=1}^{n} \mathrm{~T}_{1 \text { postfatigue }}}{\frac{1}{n} \sum_{i=1}^{n} \mathrm{~T}_{1 \text { prefatigue }}},
\end{array}
$$


and $\quad \mathrm{CFI}=\frac{\frac{1}{n} \sum_{i=1}^{n} \mathrm{VA}_{\text {postfatigue }}}{\frac{1}{n} \sum_{i=1}^{n} \mathrm{VA}_{\text {prefatigue }}}$

\section{Statistical Analyses}

We used paired $t$-tests to analyze changes before and after the 8 weeks of surface FES training. Dependent variables included MVC force, twitch force, VA, FI, PFI, and CFI. Statistical significance was set at $p<0.05$. We used the Wilcoxon signed rank test to analyze the change in MFIS score before and after the 8 weeks of surface FES training.

We used the GEE model, which accounts for correlations between observations in repeated-measures designs, to study the weighting of central fatigue and peripheral fatigue to general fatigue before and after training. The GEE model was also used to evaluate the contribution of central and peripheral fatigue resistance improvement to general fatigue resistance improvement. More specifically, Equation 5 describes the relationship between general fatigue (the decrease in MVC), central fatigue (the decrease in VA), and peripheral fatigue (the decrease in twitch force) before and after training:

$$
\mathrm{MVC}_{\mathrm{it}}=\beta_{0}+\beta_{1} \text { Twitch Force }_{\mathrm{it}}+\beta_{2} \mathrm{VA}_{\mathrm{it}}+\varepsilon_{\mathrm{it}} \text {, }
$$

where $\mathrm{MVC}_{\mathrm{it}}$ refers to observations for subject $i$ at time $t$; $\beta_{0}$ is the intercept; Twitch Force ${ }_{i t}$ and $\mathrm{VA}_{\mathrm{it}}$ are the independent variables for subject $i$ at time $t ; \beta_{1}$ and $\beta_{2}$ are the regression coefficients for independent variables twitch force and VA, respectively; and $\varepsilon_{\text {it }}$ is the "error" for subject $i$ at time $t$.

\section{RESULTS}

Seven (two males and five females, average age $42.9 \pm$ 13.5 years; all data presented as mean \pm standard deviation unless otherwise noted) out of nine subjects completed the training; two subjects did not complete the training because of disease recurrence.

After 8 weeks of surface FES training, the average knee extensor MVC increased slightly from $32.41 \pm 8.95$ kilogram weight (kgw) to $32.93 \pm 12.40 \mathrm{kgw}$, the average VA of the knee extensor increased slightly from $68.08 \pm$ 14.76 percent to $70.64 \pm 14.97$ percent, and the average twitch force increased slightly from $13.44 \pm 4.41 \mathrm{kgw}$ to $14.47 \pm 6.26 \mathrm{kgw}$, but the changes on MVC $(p=0.81)$, VA $(p=0.27)$, and twitch force $(p=0.46)$ were not statistically significant (Table 1).

The FI increased from $66.58 \pm 13.00$ percent to $74.86 \pm$ 11.02 percent $(d f=6, t=-3.77, p<0.01)$ after 8 weeks of surface FES training. This result suggests that general fatigue resistance of the patients improved (Table 1, Figure 2(a)). The CFI increased from $73.23 \pm 18.11$ percent to $84.49 \pm 11.90$ percent $(d f=6, t=-3.36, p=0.02)$ after 8 weeks of surface FES training. This result suggests that 8 weeks of surface FES training was effective in alleviating central fatigue of the patients (Table 1, Figure 2(b)). After 8 weeks of surface FES training, the average PFI changed from $93.86 \pm 7.36$ percent to $104.80 \pm 9.22$ percent, but this change was not statistically significant $(d f=6, t=-2.13$, $p=0.08$ ) (Table 1, Figure 2(c)).

Table 2 shows the results of the GEE analysis (based on Equation 5) before and after 8 weeks of training. Before 8 weeks of surface FES training, both the VA and twitch force were significantly related to MVC force. Each 1.0 unit decrease in VA led to a 0.83 unit decrease in MVC force, and each 1.0 decrease in twitch force led to a 0.15 unit decrease in MVC force. After 8 weeks of surface FES training, only VA was a significant contribution variable for MVC force. Each 1.0 unit decrease in VA led to a 0.57 unit decrease in MVC force when twitch force was controlled. This result suggests that the weighting of central fatigue is greater than the weighting of peripheral fatigue, both before and after training.

With regard to the changes before and after training, the paired-differences (post-pre) in VA and twitch force were significantly related to the paired-difference in

Table 1.

Group mean \pm standard deviation for maximum voluntary contraction (MVC), General Fatigue Index (FI), voluntary activation level (VA), Central Fatigue Index (CFI), twitch force, Peripheral Fatigue Index (PFI), and Modified Fatigue Impact Scale (MFIS) before and after 8 weeks of surface functional electrical stimulation (FES) training.

\begin{tabular}{|c|c|c|c|}
\hline Variable & Pre-FES & Post-FES & $p$-Value \\
\hline$\overline{\text { MVC (kgw) }}$ & $32.41 \pm 8.95$ & $32.93 \pm 12.40$ & 0.81 \\
\hline FI (\%) & $66.58 \pm 13.00$ & $74.86 \pm 11.02^{*}$ & $<0.01$ \\
\hline VA (\%) & $68.08 \pm 14.76$ & $70.64 \pm 14.97$ & 0.27 \\
\hline CFI (\%) & $73.23 \pm 18.11$ & $84.49 \pm 11.90^{*}$ & 0.02 \\
\hline Twitch Force (kgw) & $13.44 \pm 4.41$ & $14.47 \pm 6.26$ & 0.46 \\
\hline PFI (\%) & $93.86 \pm 7.36$ & $104.80 \pm 9.22$ & 0.08 \\
\hline MFIS (score) & $40.30 \pm 11.10$ & $34.60 \pm 14.40^{*}$ & 0.02 \\
\hline
\end{tabular}


(a)

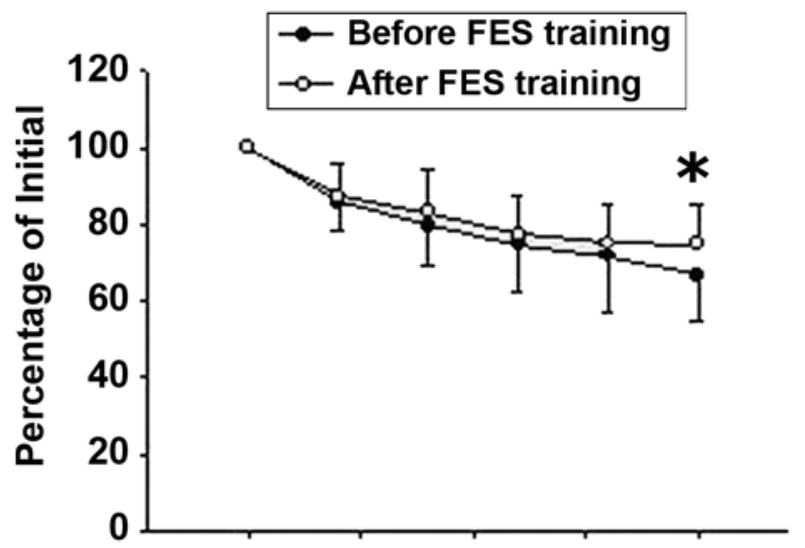

(b)

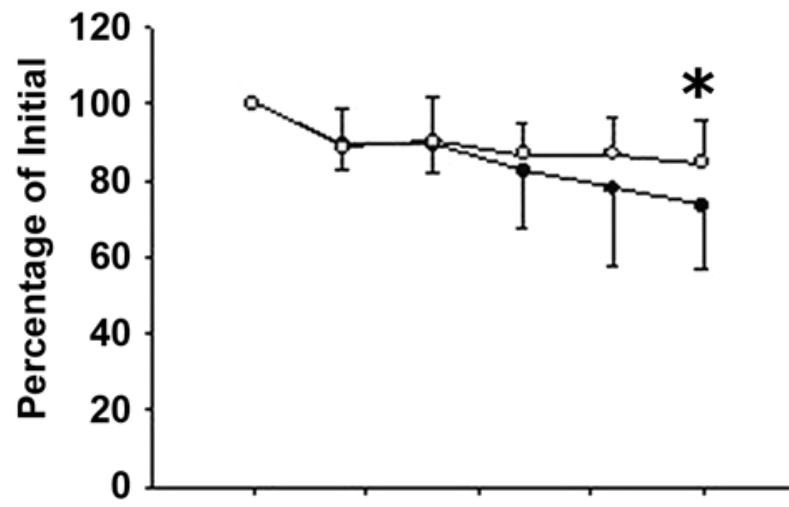

(c)

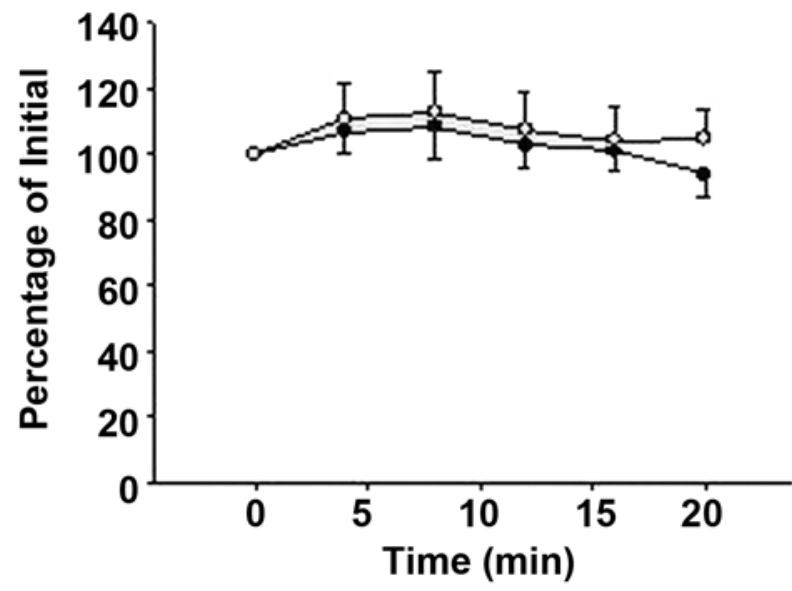

Figure 2.

Group mean \pm standard deviation of (a) normalized maximum voluntary contraction, (b) voluntary activation level, and (c) twitch force during fatiguing process before (filled circles) and after (empty circles) 8 weeks of surface functional electrical stimulation (FES) training. All values are represented as percentage of those before fatigue. Values at 20 min represent General Fatigue Index (a), Central Fatigue Index (b), and Peripheral Fatigue Index (c). * Indicates significant difference between values before and after 8 weeks of surface FES training at $20 \mathrm{~min}$.
MVC force. Each paired-difference of 1.0 unit increase in VA and each paired-difference of 1.0 unit decrease in twitch force led to the paired-difference MVC force increase of 0.53 units and decrease of 0.1 units, respectively. This result suggests that the improvement in general fatigue resistance after training was significantly related to the improvement in central fatigue.

The MFIS score decreased from $40.30 \pm 11.10$ to $34.60 \pm 14.40(S=-14, p=0.02)$ after 8 weeks of surface FES training, suggesting that patients perceived less subjective fatigue after training (Table 1).

\section{DISCUSSION}

This study is the first to quantify the weighting of central versus peripheral fatigue in MS patients. The current study showed that central fatigue was weighted more than five times higher than peripheral fatigue in individuals with MS. We also found that 8 weeks of knee-extensor surface FES training led to increases in resistance to general fatigue and resistance to central fatigue of the knee extensors in individuals with MS. The perceived fatigue measured by MFIS also improved after training. We also observed that the improvement in central fatigue resistance was the primary contributor to the improvement in general fatigue resistance after surface FES training.

We found that central fatigue contributed to the largest weighting of general fatigue and was a primary limiting factor during voluntary exercise in individuals with MS. This finding corresponds to the pathology of the disease, since demyelinating lesions occur throughout the white matter of the CNS [1]. We also found that central fatigue was weighted more than five times higher than peripheral fatigue, suggesting that the alleviation of central fatigue was important for improving general fatigue resistance. It should be noted that the current study did not disclaim the influence of peripheral fatigue on patients with MS. Lenman et al. suggested that patients with MS suffered from higher levels of peripheral fatigue than nondisabled subjects [5]. A previous study showed that in paralyzed muscle, twitch potentiated more in low frequency fatigue status than in fresh status [32]. Although they did not reach statistical significance, the results of the current study showed that the twitch force potentiated before decreasing (Figure 2(c)), suggesting that the subjects might have been in a peripheral fatigue status before the voluntary fatigue protocol was applied. 
Table 2.

Summary of results of generalized estimating equations analysis. "Pre” indicates regression coefficients before surface functional electrical stimulation (FES) training. "Post” indicates regression coefficients after 8 weeks of surface FES training. Post-Pre indicates pairwise change for regression coefficients before and after 8 weeks of training. VA and TW are shown as percentage of initial before fatigue.

\begin{tabular}{|c|c|c|c|c|}
\hline Variable & Regression Coefficient & Standard Error & 95\% Confidence Limit & $p$-Value \\
\hline \multicolumn{5}{|l|}{ Pre } \\
\hline Intercept & -7.12 & 5.98 & $-18.85,4.61$ & 0.23 \\
\hline VA & 0.83 & 0.07 & $0.70,0.96$ & $<0.01$ \\
\hline \multicolumn{5}{|l|}{ Post } \\
\hline Intercept & 24.41 & 10.79 & $3.27,45.56$ & 0.02 \\
\hline \multicolumn{5}{|l|}{ Post-Pre } \\
\hline Intercept & 3.51 & 1.27 & $1.01,6.00$ & 0.01 \\
\hline VA & 0.53 & 0.14 & $0.27,0.80$ & $<0.01$ \\
\hline TW & -0.10 & 0.04 & $-0.19,-0.02$ & 0.02 \\
\hline
\end{tabular}

Whether the peripheral fatigue was caused by routine daily activities and why the full recovery did not occur remain unclear.

The current study is the first to report that surface FES could improve central fatigue in individuals with MS. Vahtera et al. used electrical stimulation to strengthen the pelvic floor muscle in patients with MS and found that EMG activities increased following the training [33], but the effect of central fatigue was not evaluated. Previous studies reported that electrical stimulation of peripheral nerves at an intensity above the motor threshold increased the transcranial magnetic stimulation and elicited motor evoked potential without a change in H-reflex, suggesting that electrical stimulation alone could induce motor cortex plasticity [21-22,34-36]. In the current study, the intensity of surface FES was higher than the motor threshold and, thus, was considered to be able to induce motor cortex plasticity. Such an increase in plasticity might alleviate central fatigue in individuals with MS. Consequently, general fatigue was improved as a result of the decreased central fatigue.

Peripheral muscle strengthening is one of the important effects of FES $[18,20]$. Peripheral muscle strengthening alone might lead to an improvement in central fatigue. In a pilot study, Mount and Dacko found that 8 weeks of peripheral muscle strengthening by isometric voluntary contractions increased endurance [37]. Although the correlation between endurance and central fatigue was not significant, they found that one of the subjects with a significant improvement in endurance had a significant decrease in central activation failure. Mount and Dacko suggested that peripheral muscle strengthening provided a chance for the CNS to learn [37]. Although FES bypasses the CNS and the motor command pathway is different from that in voluntary contractions, FES elicits muscle contractions similar to voluntary contractions and increases the afferent input to the cortex, which might provide a chance for the CNS to learn and thereby decrease central fatigue.

The improvement in general fatigue following surface FES training was primarily the result of the improvement of central fatigue resistance. The current study found that a 1.0 unit increase in the paired-difference of VA caused the paired-difference of MVC to increase by 0.53 units, while a 1.0 unit decrease in the paired-difference of twitch force caused the paired-difference of MVC to decrease by 0.1 units. Furthermore, peripheral fatigue did not change significantly after 8 weeks of surface FES training. Gerrits et al. found that 12 weeks of electrical stimulation training in individuals with chronic spinal cord injury improved the fatigue index and increased the percentage of slow fibers in the quadriceps muscle [38-39].

Gondin et al. suggested that neural adaptation occurred primarily during the first 4 weeks of training, whereas changes in muscle mass were detected after 4 to 8 weeks of training [40]. In the current study, the stimulation intensity during the first 2 weeks was less than the targeted intensity required for subjects to accommodate the electrical stimulation. The optimal training intensity was not attained until the third week of training. The current 
study failed to show significant improvements in twitch force and PFI, likely because of an insufficient number of training sessions at the optimal training intensity required to induce changes in muscle mass. Further studies with more training sessions at the optimal intensity might be able to show significant improvements in twitch force and/or PFI.

The current study showed that the MFIS score decreased after 8 weeks of surface FES training. This result suggests that patients with MS felt that the 8 weeks of training were beneficial. This is the first study to show that surface FES training decreased the perception of fatigue in patients with MS. Research has shown that resistance training can decrease the perception of fatigue in patients with MS [10]. In comparison to resistance training, surface FES training requires less active participation and, thus, may be appropriate for patients with MS who are restricted from performing high-intensity voluntary exercise and/or who usually fatigue before reaching an optimal level of voluntary resistance training.

\section{LIMITATIONS}

The patients included in this study had EDSS scores between 1.0 and 4.0. The effect of 8 weeks of surface FES training on patients with MS who have severe motor deficits is not known. However, we believe that patients with MS with more severe motor deficits may benefit more from surface FES training than those who have minor motor deficits. We did not include a control group in this study because it is difficult to identify a medical condition with physical functions that match those of patients with MS. We did not use the subjects' opposite limb as a control because it is not an appropriate control, especially for VAs and central fatigue measurements. A common concern with before-after study design is the change in health status of the subjects during the study period, although this was not the case for the current study. The medical conditions and lifestyles of the subjects in the current study remained the same during the training sessions.

\section{CONCLUSIONS}

Central fatigue is weighted more than five times higher than peripheral fatigue in individuals with MS. Eight weeks of surface FES training led to improvements in general fatigue, central fatigue, and perception of fatigue in MS patients. The decrease in general fatigue after surface FES training was associated with a decrease in central fatigue. A home-based surface FES training program, which requires less equipment and less active participation by patients with MS, is recommended for those who have difficulty participating in voluntary training programs.

\section{CLINICAL IMPLICATIONS}

- Central fatigue is a primary limitation during voluntary exercise of individuals with MS. The peripheral neuromuscular system might not receive a sufficient level of training during voluntary exercise programs.

- Home-based surface FES, which does not require activation of the CNS, is a safe and effective training strategy for individuals with MS.

- Eight weeks of surface FES can reduce general fatigue, decrease central fatigue, and lower perceived fatigue.

\section{ACKNOWLEDGMENTS}

\section{Author Contributions:}

Study concept and design: Y. J. Chang, M. J. Hsu, S. M. Chen, C. H. Lin, A. M. K. Wong.

Acquisition of data: Y. J. Chang, S. M. Chen.

Analysis and interpretation of data: Y. J. Chang, M. J. Hsu, C. H. Lin, A. M. K. Wong.

Drafting of manuscript: Y. J. Chang, M. J. Hsu, S. M. Chen, C. H. Lin, A. M. K. Wong.

Critical revision of manuscript for important intellectual content:

Y. J. Chang, M. J. Hsu, S. M. Chen, C. H. Lin, A. M. K. Wong.

Statistical analysis: Y. J. Chang, C. H. Lin.

Obtained funding: Y. J. Chang, A. M. K. Wong.

Study supervision: Y. J. Chang, M. J. Hsu, C. H. Lin, A. M. K. Wong.

Financial Disclosures: The authors have declared that no competing interests exist.

Funding/Support: This material was based on work supported by the National Science Council, Taiwan (grant NSC 95-2314-B-182-044MY2).

Institutional Review: The study was approved by the Chang Gung Medical Foundation Institutional Review Board. All subjects gave written informed consent in accordance with the Declaration of Helsinki.

Participant Follow-Up: Subjects who participated in this study will be notified of study publication. 


\section{REFERENCES}

1. O’Sullivan SB, Schmitz TJ. Physical rehabilitation: Assessment and treatment. 4th ed. Philadelphia (PA): F.A. Davis; 2001. p. 715-36.

2. Gorelick PB. Clues to the mystery of multiple sclerosis. Postgrad Med. 1989;85(4):125-28,131-34. [PMID: 2648350]

3. Kent-Braun JA, Sharma KR, Miller RG, Weiner MW. Postexercise phosphocreatine resynthesis is slowed in multiple sclerosis. Muscle Nerve. 1994;17(8):835-41.

[PMID: 8041390]

DOI:10.1002/mus.880170802

4. Ng AV, Miller RG, Gelinas D, Kent-Braun JA. Functional relationships of central and peripheral muscle alterations in multiple sclerosis. Muscle Nerve. 2004;29(6):843-52.

[PMID: 15170617] DOI:10.1002/mus.20038

5. Lenman AJ, Tulley FM, Vrbova G, Dimitrijevic MR, Towle JA. Muscle fatigue in some neurological disorders. Muscle Nerve. 1989;12(11):938-42. [PMID: 2608089] DOI:10.1002/mus.880121111

6. Berg HE, Dudley GA, Hather B, Tesch PA. Work capacity and metabolic and morphologic characteristics of the human quadriceps muscle in response to unloading. Clin Physiol. 1993;13(4):337-47. [PMID: 8370234]

DOI:10.1111/j.1475-097X.1993.tb00334.X

7. Liang K, Zeger SL. Longitudinal data analysis using generalized linear models. Biometrika. 1986;73(1):13-22. DOI:10.1093/biomet/73.1.13

8. Zeger SL, Liang KY. Longitudinal data analysis for discrete and continuous outcomes. Biometrics. 1986;42(1):121-30. [PMID: 3719049] DOI:10.2307/2531248

9. Chang YJ, Liu CC, Lin CH, Tsaih PL, Hsu MJ. Using electromyography to detect the weightings of the local muscle factors to the increase of perceived exertion during stepping exercise. Sensors. 2008;8(6):3643-55. DOI:10.3390/s8063643

10. Svensson B, Gerdle B, Elert J. Endurance training in patients with multiple sclerosis: Five case studies. Phys Ther. 1994;74(11):1017-26. [PMID: 7972362]

11. DeBolt LS, McCubbin JA. The effects of home-based resistance exercise on balance, power, and mobility in adults with multiple sclerosis. Arch Phys Med Rehabil. 2004;85(2):290-97. [PMID: 14966716]

DOI:10.1016/j.apmr.2003.06.003

12. Gehlsen GM, Grigsby SA, Winant DM. Effects of an aquatic fitness program on the muscular strength and endurance of patients with multiple sclerosis. Phys Ther. 1984;64(5):653-57. [PMID: 6718494]

13. Kileff J, Ashburn A. A pilot study of the effect of aerobic exercise on people with moderate disability multiple sclero- sis. Clin Rehabil. 2005;19(2):165-69. [PMID: 15759531]

DOI:10.1191/0269215505cr839oa

14. Petajan JH, Gappmaier E, White AT, Spencer MK, Mino L, Hicks RW. Impact of aerobic training on fitness and quality of life in multiple sclerosis. Ann Neurol. 1996;39(4):432-41. [PMID: 8619521]

DOI:10.1002/ana.410390405

15. Ponichtera-Mulcare JA, Mathews T, Barrett PJ, Glaser RM. Change in aerobic fitness of patients with multiple sclerosis during a 6-month training program. Sports Med Train Rehabil. 1997;7:265-72.

16. Ponichtera-Mulcare JA, Mathews T, Glaser RM, Gupta SC. Maximal aerobic exercise of individuals with multiple sclerosis using three modes of ergometry. Clin Kinesiol. 1995;49(1): 4-12.

17. Gallien P, Nicolas B, Robineau S, Pétrilli S, Houedakor J, Durufle A. Physical training and multiple sclerosis. Ann Readapt Med Phys. 2007;50(6):373-6,369-72. [PMID: 17482708]

18. Abdel-Moty E, Fishbain DA, Goldberg M, Cutler R, Zaki AM, Khalil TM, Peppard T, Rosomoff RS, Rosomoff HL. Functional electrical stimulation treatment of postradiculopathy associated muscle weakness. Arch Phys Med Rehabil. 1994;75(6):680-86. [PMID: 8002769]

DOI:10.1016/0003-9993(94)90194-5

19. Embrey DG, Holtz SL, Alon G, Brandsma BA, McCoy SW. Functional electrical stimulation to dorsiflexors and plantar flexors during gait to improve walking in adults with chronic hemiplegia. Arch Phys Med Rehabil. 2010;91(5): 687-96. [PMID: 20434604]

DOI:10.1016/j.apmr.2009.12.024

20. Quittan M, Sochor A, Wiesinger GF, Kollmitzer J, Sturm B, Pacher R, Mayr W. Strength improvement of knee extensor muscles in patients with chronic heart failure by neuromuscular electrical stimulation. Artif Organs. 1999; 23(5):432-35. [PMID: 10378936] DOI:10.1046/j.1525-1594.1999.06372.X

21. Chang YJ, Hsieh TH, Huang YM, Hsu MJ, Wong AMK. A lack of modulation of motor evoked potential in sensoryimpaired individuals with spinal cord injuries. J Med Biol Eng. 2011;31(1):37-44.

22. Ridding MC, McKay DR, Thompson PD, Miles TS. Changes in corticomotor representations induced by prolonged peripheral nerve stimulation in humans. Clin Neurophysiol. 2001;112(8):1461-69. [PMID: 11459686$]$ DOI:10.1016/S1388-2457(01)00592-2

23. Kurtzke JF. Rating neurologic impairment in multiple sclerosis: An Expanded Disability Status Scale (EDSS). Neurology. 1983;33(11):1444-52. [PMID: 6685237]

24. Yue GH, Ranganathan VK, Siemionow V, Liu JZ, Sahgal V. Evidence of inability to fully activate human limb muscle. Muscle Nerve. 2000;23(3):376-84. [PMID: 10679714$]$ 
DOI:10.1002/(SICI)1097-4598(200003)23:3<376::AIDMUS9>3.0.CO;2-2

25. Lloyd AR, Gandevia SC, Hales JP. Muscle performance, voluntary activation, twitch properties and perceived effort in normal subjects and patients with the chronic fatigue syndrome. Brain. 1991;114(Pt 1A):85-98. [PMID: 1998892]

26. Mademli L, Arampatzis A. Effect of voluntary activation on age-related muscle fatigue resistance. J Biomech. 2008; 41(6):1229-35. [PMID: 18342865]

DOI:10.1016/j.jbiomech.2008.01.019

27. Belanger AY, McComas AJ. Extent of motor unit activation during effort. J Appl Physiol. 1981;51(5):1131-35.

[PMID: 7298453]

28. Huang YM, Hsu MJ, Lin CH, Wei SH, Chang YJ. The nonlinear relationship between muscle voluntary activation level and voluntary force measured by the interpolated twitch technique. Sensors. 2010;10(1):796-807. DOI:10.3390/s100100796

29. Li JL, Wang XN, Fraser SF, Carey MF, Wrigley TV, McKenna MJ. Effects of fatigue and training on sarcoplasmic reticulum $\mathrm{Ca}(2+)$ regulation in human skeletal muscle. J Appl Physiol. 2002;92(3):912-22. [PMID: 11842021]

30. Binder-Macleod SA, Snyder-Mackler L. Muscle fatigue: Clinical implications for fatigue assessment and neuromuscular electrical stimulation. Phys Ther. 1993;73(12):902-10. [PMID: 8248298]

31. Yoon T, Schlinder Delap B, Griffith EE, Hunter SK. Mechanisms of fatigue differ after low- and high-force fatiguing contractions in men and women. Muscle Nerve. 2007; 36(4):515-24. [PMID: 17626289$]$ DOI:10.1002/mus.20844

32. Chang YJ, Shields RK. Within-train neuromuscular propagation varies with torque in paralyzed human muscle. Muscle Nerve. 2002;26(5):673-80. [PMID: 12402290] DOI:10.1002/mus.10245

33. Vahtera T, Haaranen M, Viramo-Koskela AL, Ruutiainen J. Pelvic floor rehabilitation is effective in patients with multiple sclerosis. Clin Rehabil. 1997;11(3):211-19.

[PMID: 9360033] DOI:10.1177/026921559701100304

34. Kaelin-Lang A, Luft AR, Sawaki L, Burstein AH, Sohn YH, Cohen LG. Modulation of human corticomotor excitability by somatosensory input. J Physiol. 2002;540(Pt 2):623-33. [PMID: 11956348]

DOI:10.1113/jphysiol.2001.012801
35. Knash ME, Kido A, Gorassini M, Chan KM, Stein RB. Electrical stimulation of the human common peroneal nerve elicits lasting facilitation of cortical motor-evoked potentials. Exp Brain Res. 2003;153(3):366-77.

[PMID: 14610631]

DOI:10.1007/s00221-003-1628-9

36. Yang H. Facilitation of the motor evoked potentials by median nerve stimulation in patients with spinocerebellar ataxia [unpublished master's thesis]. [Taiwan]: Chang Gung University; 2005.

37. Mount J, Dacko S. Effects of dorsiflexor endurance exercises on foot drop secondary to multiple sclerosis: A pilot study. NeuroRehabilitation. 2006;21(1):43-50.

[PMID: 16720937]

38. Gerrits HL, De Haan A, Hopman MT, Van Der Woude LH, Jones DA, Sargeant AJ. Contractile properties of the quadriceps muscle in individuals with spinal cord injury. Muscle Nerve. 1999;22(9):1249-56. [PMID: 10454722] DOI:10.1002/(SICI)1097-4598(199909)22:9<1249::AIDMUS13>3.0.CO;2-N

39. Gerrits HL, Hopman MT, Sargeant AJ, Jones DA, De Haan A. Effects of training on contractile properties of paralyzed quadriceps muscle. Muscle Nerve. 2002;25(4):559-67. [PMID: 11932974] DOI:10.1002/mus.10071

40. Gondin J, Guette M, Ballay Y, Martin A. Electromyostimulation training effects on neural drive and muscle architecture. Med Sci Sports Exerc. 2005;37(8):1291-99.

[PMID: 16118574]

DOI:10.1249/01.mss.0000175090.49048.41

Submitted for publication March 17, 2010. Accepted in revised form November 23, 2010.

This article and any supplementary material should be cited as follows:

Chang YJ, Hsu MJ, Chen SM, Lin CH, Wong AMK. Decreased central fatigue in multiple sclerosis patients after 8 weeks of surface functional electrical stimulation. J Rehabil Res Dev. 2011;48(5):555-64.

DOI:10.1682/JRRD.2010.03.0038

ResearcherID: Ya-Ju Chang, PhD: B-9326-2011. 